\title{
Assessing feasibility and acceptability of a brief intervention for risky alcohol consumption in sexual health clinic attendees: a randomised controlled trial
}

\author{
Paul Roderick, ${ }^{1}$ Sangeetha S Sundaram, ${ }^{2}$ Borislav D Dimitrov, ${ }^{1}$ \\ Susan Dewhirst, ${ }^{1}$ Linda J Tucker, ${ }^{2}$ Geraldine Leydon, ${ }^{1}$ Nick Sheron, ${ }^{3}$ \\ Alison Frater, ${ }^{4}$ Veerakathy Harindra ${ }^{2}$
}

\begin{abstract}
- Additional material is published online only. To view please visit the journal online (http://dx.doi.org/10.1136/jprhc2014-100912)

For numbered affiliations see end of article.

\section{Correspondence to} Professor Paul Roderick, Academic Unit of Primary Care \& Population Sciences, C Floor, South Academic Block, University Hospital Southampton, Tremona Road, Southampton S016 6YD, UK; pjr@soton.ac.uk
\end{abstract}

Received 21 February 2014 Revised 10 June 2015 Accepted 1 July 2015 Published Online First 10 August 2015

CrossMark

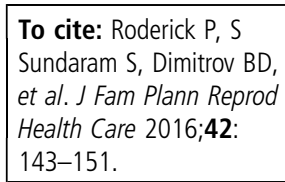

\begin{abstract}
Objectives To assess the feasibility and acceptability of screening attendees at a sexual health clinic (SHC) for alcohol misuse, and delivering a brief intervention (BI). To explore the effect of this $\mathrm{BI}$ on drinking and sexual behaviour. Methods A consecutive sample of consenting SHC attendees aged $\geq 16$ years were screened using Alcohol Use Disorders Identification Test Consumption (AUDIT-C). Men scoring $\geq 5$ and women scoring $\geq 4$ were invited to complete the full AUDIT, alcohol diary and baseline questionnaire.

Interventions Participants were randomised to receive $\mathrm{BI}$ by a trained sexual health professional or a standard alcohol leaflet (usual care, UC).

All were followed up for changes in alcohol and sexual behaviour at 6 weeks and 6 months.

A fidelity check and staff focus group were undertaken.
\end{abstract}

Results Of 664 participants screened, 215 (32\%) were eligible for randomisation and 207 were included in the final analysis: 103 (BI) and 104 (UC). Follow-up rates were $54 \%$ and $47 \%$ at 6 weeks and 6 months, respectively. Both groups reduced alcohol consumption though the degree of change did not differ between them. There was some evidence of positive changes in sexual health risk in both groups. BI was delivered as intended, adding 5 minutes to the consultation, and staff feedback was positive.

Conclusions Alcohol misuse was common in SHC attendees. Systematic assessment and BI for alcohol misuse was feasible and acceptable to staff and patients. Identification and provision of standard information alone appeared to influence drinking and sexual behaviour.

Trial registration number ISRCTN19452424.

\section{Key message points}

- It is feasible to assess alcohol use and deliver a brief intervention in a sexual health setting; follow-up rate in this target group was not high.

- Screening followed by simple written information alone appeared to influence drinking and sexual behaviour in this trial.

- Further research is needed to develop and evaluate an intervention for the heaviest drinkers and to evaluate this approach in other sexual health settings.

\section{INTRODUCTION}

Excessive drinking is a social problem in the UK, with binge drinking in young people being a key part of the problem. ${ }^{1}$ In 2009, a quarter of adults aged 16 years and over in England (22\%) drank above recommended limits, and about one in six reported binge drinking. ${ }^{2}$ Rising alcohol-related mortality and morbidity are well documented including organ damage (e.g. liver cirrhosis), psychiatric morbidity, trauma, unintended pregnancy and sexually transmitted infections (STIs). ${ }^{3-5}$ Rates of STIs are highest in young people. ${ }^{5}$ Alcohol use and sexual behaviour are closely linked (e.g. alcohol may enhance perceived confidence in relationships but may lead to unsafe $\left.\operatorname{sex}^{6}\right)$. A systematic review on the 
association between 'problem drinking' and adverse sexual health identified 11 studies of which eight found a positive association, though proving causation was limited by methodology such as small sample size, poor definition of problem drinking, sexual health outcomes and cross-sectional designs. ${ }^{7}$ A study in Portsmouth, UK found high alcohol use among sexual health clinic (SHC) attendees; 86\% reported binge drinking with mean consumption in excess of 20 units (a 'unit' is a standard measure of alcohol equivalent to $8 \mathrm{~g}$ or $10 \mathrm{ml}$ of pure alcohol) on a 'heavy' night. ${ }^{8}$ Level of alcohol use in this study was positively associated with number of sexual partners and unintended pregnancy. Few had received education on alcohol use from school or other sources.

Early identification and brief intervention (BI) is increasingly advocated as a cost-effective strategy to reduce problem drinking. To date the strongest evidence is in primary care. A systematic Cochrane Review of 29 primary care trials reported that BI was associated with a significant reduction in alcohol consumption, equivalent to 4-5 units/week in comparison to controls receiving screening only, treatment as usual or written information. ${ }^{9}$ Most of these trials have focused on middle-aged drinkers with other groups under-represented. Common features of BI were personalised feedback on alcohol use and related harm including the benefits of reducing consumption, motivational enhancement, analysis of high-risk situations for problem drinking and coping strategies.

Limited data exist on the feasibility of integrating an alcohol BI into the busy SHC consultation. An Australian study found a reduction in alcohol intake using the Alcohol Use Disorders Identification Test (AUDIT) score in both nurse-delivered BI and control groups over a 3-month follow-up and reduction in binge drinking in their BI group; sexual health outcomes were not assessed. ${ }^{10}$ The National Institute of Health and Care Excellence (NICE) recommends routine screening for alcohol-use disorders in genitourinary medicine clinics as individuals who regularly attend them may be at increased risk of alcoholrelated harm. ${ }^{11}$

This study aimed to assess the feasibility and acceptability of screening for alcohol misuse, randomisation and delivery of a BI to adults attending a large, urban, inner-city SHC. We also aimed to explore the effect of $\mathrm{BI}$ on alcohol and sexual behaviour over a 6-month follow-up period.

\section{METHODOLOGY}

This was a two parallel-arm randomised controlled trial (RCT) with a qualitative component.

\section{Study setting}

The study was conducted in a large, inner-city SHC in one of the most deprived areas of Portsmouth City. This clinic has good transport links to the city centre and is accessible to young people including student populations. The department saw over 30000 attendees per year (2010-2011) of which 17000 were new attendances, approximately $40 \%$ having proven STIs.

\section{Inclusion and exclusion criteria}

All attendees aged 16 years and over, irrespective of their sexual orientation or gender, attending the clinic for the first episode of care, were recruited. Both symptomatic and asymptomatic presentations were included. Recruitment took place over 7 months from April to October 2011.

\section{Recruitment}

Reception staff handed out a study information sheet [which outlined the aims of the study and the purpose of the Alcohol Use Disorders Identification Test Consumption (AUDIT-C)], a consent form and the three-item AUDIT-C ${ }^{12}$ card. Participants who consented for the study completed the AUDIT-C and handed it back to the receptionist. The AUDIT-C scores were calculated by a researcher. Men scoring $\geq 5$ and women scoring $\geq 4$ were invited to complete a questionnaire which included the following:

- Sociodemographic information (education, employment, ethnicity)

- The 10-item AUDIT questionnaire ${ }^{13} 14$

- Retrospective alcohol diary to elicit patterns of consumption over the last week, amount/day on the heaviest drinking day (HDD) (defined by Office for National Statistics as $>4$ units for men and $>3$ units for women) and on a usual day in the last month

- Smoking and drug use

- Perceptions of problem drinking and measure of self-efficacy

- Sexual behaviour (i.e. change in partners, number of partners and condom use with new partners).

Information about the reason for attendance at the SHC was collected from the participants' routine clinical records.

Participants with a high AUDIT score $>15$ suggesting alcohol dependence were excluded from the study and flagged up for clinic staff to be referred either to their general practitioner or directly to the Portsmouth Alcohol Intervention Team (AIT). This criterion was later relaxed during the study and the threshold was raised to $>20$ to avoid missing a key target group with AUDIT scores of 15-20.

\section{Randomisation}

Participants were randomised to one of two arms: BI or usual care (UC) in blocks of variable size (four, six and eight that were randomly selected). BI and UC were randomly allocated, whilst maintaining a 50:50 ratio within each block. The overall sequence was contained in a series of individually numbered and sealed envelopes that were held in the clinic. In this single centre, only clinics with more than two staff trained 
in BI were used. Block randomisation ensured that any chosen clinic session could manage patient recruitment.

\section{Brief intervention}

BI was delivered by one of a team of five trained sexual health staff (four nurses and a non-consultant grade doctor) as part of the routine clinical consultation. These members of staff were randomised to receive training in delivering BI or not by stratified randomisation to avoid volunteer bias. All attended two sessions of training given by the Portsmouth City Primary Care Trust AIT. The BI, using the FRAMES ${ }^{15}$ (Feedback, Responsibility, Advice, Menu, Empathy, Self-efficacy) approach consisted of:

- Feedback on participants' drinking levels compared with national norms and peers

- Giving information about potential harms and negative social consequences of alcohol

- Making a link between alcohol and its contribution to the presenting sexual health problem

- Discussion of techniques outlining means for reducing consumption (e.g. avoiding situations that usually result in heavy drinking) focusing on personal responsibility

- Written information offered as a leaflet designed for this study 'Sensible drinking: Enjoy your drink and know what it could be doing to your body and mind' AND a copy of the patient information leaflet on 'Alcohol and sensible drinking' (http:/www.patient.co.uk/health/ Alcohol-and-Sensible-Drinking.html).

\section{Usual care}

Participants randomised to the UC arm were seen by sexual health staff (nurses and non-consultant grade doctors) not trained in BI. They were only offered a copy of the patient information leaflet on Alcohol and sensible drinking'.

\section{Follow-up}

We attempted to contact all patients at 6 weeks and at 6 months. A $£ 10$ cinema voucher was offered as an incentive for participants to complete follow-up at both time points. Initially we tried to undertake 6-week follow-up by texting and asking individuals to self-complete a questionnaire on a secure internet website hosted at the University of Southampton, with a unique personal access code. However, there was low uptake so we switched to text reminders and telephone contact for both follow-up periods. We phoned up to three times in order to make contact and undertook the follow-up questionnaire (which was also used at baseline) by telephone, blinded from the participant's allocation status wherever possible. If we could not make contact by phone we sent a single postal questionnaire. The schedule included the AUDIT questionnaire (6 months only), alcohol diary and specific questions on alcohol consumption on a usual day and HDD, history of alcohol-related harm (i.e. accident and emergency department attendances, hospitalisation) (6 months only) and standard questions on sexual risk behaviour. Clinic records were reviewed at 6 months for new episodes of attendance, reasons for attendance and evidence of any new STIs.

\section{Statistical analysis}

Information transcribed from the AUDIT-C card, questionnaires and clinic data were entered onto a secure database and analysed using SAS (SAS Institute Inc.) and SPSS (IBM) software packages. The intervention status was blinded for data entry and analysis. We analysed change over time by completed cases (data shown) and also by assuming no change from baseline in non-responders (data not shown). Standard descriptive methods were used with frequency $(\%)$ and mean [ \pm standard deviation (SD)] statistics. The type of the distributions of quantitative variables was checked by the Shapiro-Wilk test. Since only few of the variables had a normal distribution, the results were presented also as median and interquartile range (IQR) unless stated otherwise. The study was not powered to assess changes over time between the groups, so we did not undertake formal statistical comparisons. Hazardous/harmful drinking was defined as $>14$ units/week in women and $>21$ units/week in men. ${ }^{16}$

\section{Fidelity check}

A sample of BI and UC consultations were audiorecorded and structured analysis assessed the fidelity of the BI and UC consultation. For BI this entailed listening to all consultations to complete a checklist of elements of the intended BI. Alcohol talk in the UC consultations was also checked and the timing and length of the talk noted.

Acceptability to staff was evaluated using a single focus group of the trained BI staff. The focus group was facilitated by two skilled qualitative researchers. A topic guide was used to explore staff views on what worked well and what could have worked better with the study and BI. The group was audio-recorded and transcribed verbatim in preparation for thematic analysis drawing on Braun and Clarke's thematic phases. ${ }^{17}$ The computer software package Nvivo 9.2 ${ }^{\mathrm{TM}}$ (QSR International) was used to organise the data.

\section{RESULTS}

Of the 664 participants who were given an AUDIT-C card at clinic reception, 556 (84\%) participants returned a completed AUDIT-C card. Of these, 190 (34\%) were not eligible to enter the study due to low AUDIT-C scores. Of the 362 participants who were eligible and completed the full AUDIT, 63 were in the high AUDIT group (score $>15$ ) and 84 declined after providing initial consent. A clinician had already seen two patients; one was given an AUDIT-C in error and one was not able to read the study information. 
This left 215 participants for randomisation: 107 in BI arm, 108 in UC arm (Figure 1). The main reasons for loss of numbers were non-completion of AUDIT-C, a low AUDIT-C score, and patient eligible but declined. Eight people (four in each arm) did not complete baseline information, leaving 207 with baseline data: $103 \mathrm{BI}$ and $104 \mathrm{UC}$.

\section{Baseline characteristics}

The mean age was 25 years, $66 \%$ were female, most were White, nearly $50 \%$ were in full-time employment and $27 \%$ were students. Alcohol consumption was similar between the two groups. There was some imbalance between the two groups with UC patients more likely to be female, students and attending for asymptomatic advice with a higher frequency of sexual activity (Table 1). The overall AUDIT score was 10.1 (SD 3.6) and was similar between the two groups. Some 56\% were drinking hazardously/harmfully.

\section{Follow-up}

Follow-up at 6 weeks and 6 months was $54 \%$ and $47 \%$, respectively. Follow-up questionnaires at both time points were largely completed by telephone interview with study researchers. There was no

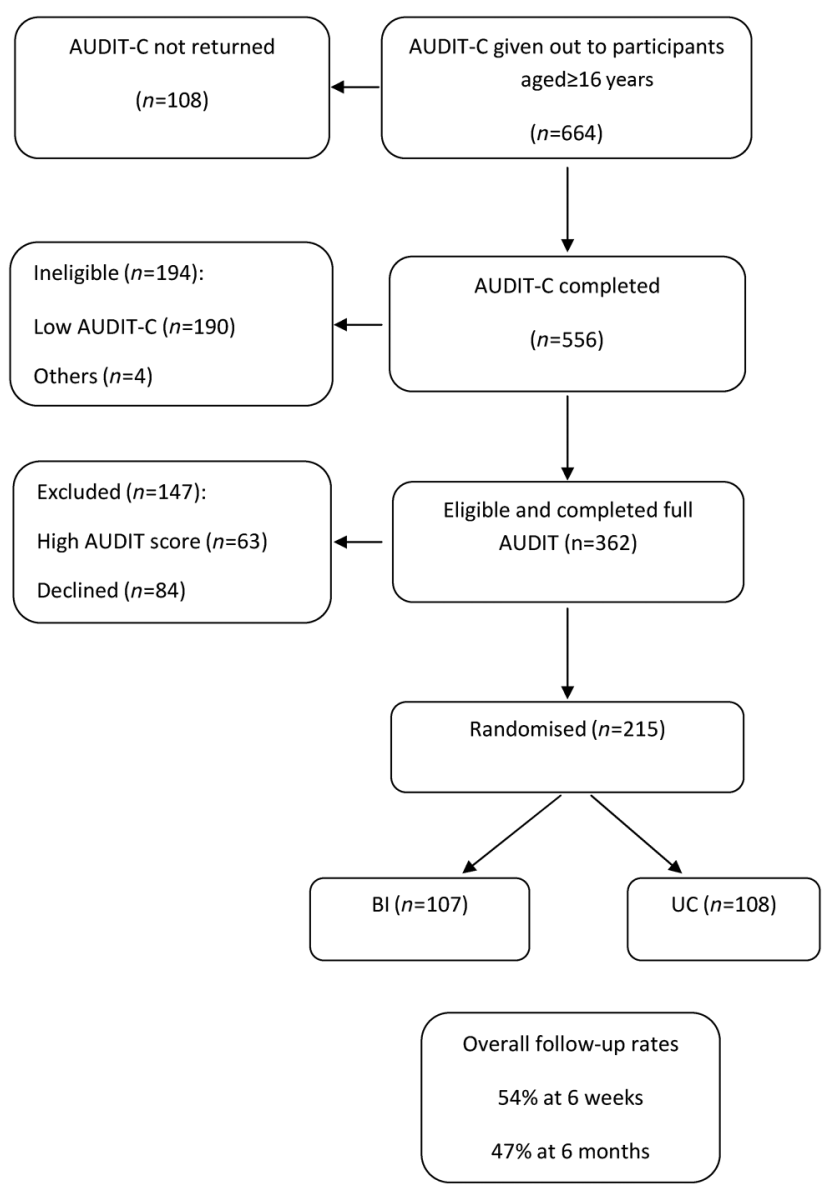

Figure 1 CONSORT diagram of trial profile. AUDIT-C, Alcohol Use Disorders Identification Test Consumption; Bl, brief intervention; UC, usual care. difference in age or sex by response category; however, in the UC arm responders were more likely to be heavier drinkers (in terms of units/week, AUDIT score, HDD) at baseline (see Online Supplementary Material Appendix 1).

\section{Alcohol changes}

On complete case analysis $(n=98)$ both groups tended to reduce their alcohol consumption across different measures except units drunk on a typical day but as there was reduction in days drinking, overall units per week fell (Table 2). Fewer participants reported drinking hazardously or harmfully following recruitment and most of the reported changes had occurred by 6 weeks. For most measures there were no striking between-group differences, except total alcohol units/ week fell more in UC using both complete case analysis (i.e. responders). Any outcome change should be treated with caution due to the small numbers as we were not powered for effect differences and imbalances between the groups existed at baseline and follow-up. Changes in alcohol use tended to be greater in the 28 patients we recruited with baseline AUDIT scores $>15$ (data not shown). Very few had advice on alcohol use after recruitment from a source other than the SHC.

\section{Sexual health changes}

There was no evidence of difference in sexual behaviour between the groups at any time point, though some evidence of change over time overall in 'partner frequency' and in 'regretted sex' after alcohol in both arms (Table 3). There was no difference in the frequency of return to the SHC for further episodes.

\section{Fidelity check}

A sample of $21 \mathrm{BI}$ and $8 \mathrm{UC}$ consultations were analysed using a structured framework to determine the impact of BI on consultation length and to assess fidelity. Sampling ensured that audio-recordings of all staff trained in BI were reviewed. The average time of the UC and BI consultations were 8.28 and 12.8 minutes, respectively. Indeed almost 5 minutes were spent talking about alcohol in the BI consultations sampled.

The aspects of BI most commonly discussed were units of alcohol $(n=19)$, the risks of drinking $(n=19)$, targets for reducing alcohol consumption $(n=19)$, plan for reducing $(n=18)$, use of diary $(n=17)$ and leaflet $(n=16)$. The link between alcohol and sexual health was discussed in 10 consultations and the benefits to sexual health of reducing consumption in 13 consultations.

\section{Acceptability of BI to clinic staff}

Focus group analysis showed that overall staff were pleased to take part in the study and were supportive of this kind of intervention in the SHC setting. There was agreement that most of the participants were not 
Table 1 Baseline characteristics - all cases $(n=207)$

\begin{tabular}{|c|c|c|c|}
\hline Parameters* & Usual care $(n=104)$ & Brief intervention $(n=103)$ & Total $(n=207)$ \\
\hline \multicolumn{4}{|l|}{ Sociodemographic characteristics } \\
\hline Age (years) & $24.3 \pm 7.6(22,19-27)$ & $25.7 \pm 8.0(23,20-28)$ & $25.0 \pm 7.8(22,20-28)$ \\
\hline \multicolumn{4}{|l|}{ Gender } \\
\hline Female & $74(71.8)$ & $62(60.2)$ & $136(66.0)$ \\
\hline Male & $29(28.2)$ & $41(39.8)$ & $70(34.0)$ \\
\hline \multicolumn{4}{|l|}{ Ethnicity } \\
\hline White & $101(97.1)$ & $95(92.2)$ & $196(94.7)$ \\
\hline Other & $3(2.9)$ & $8(7.8)$ & $11(5.3)$ \\
\hline \multicolumn{4}{|l|}{ Marital status } \\
\hline Single & $97(93.3)$ & $89(86.4)$ & $186(89.9)$ \\
\hline Married & $2(1.9)$ & $3(2.9)$ & $5(2.4)$ \\
\hline Other & $5(4.8)$ & $11(10.7)$ & $16(7.7)$ \\
\hline \multicolumn{4}{|l|}{ Employment status } \\
\hline Full-time & $47(45.20)$ & $53(51.5)$ & $100(48.3)$ \\
\hline Part-time & $15(14.42)$ & $17(16.5)$ & $32(15.5)$ \\
\hline Unemployed & $7 \quad(6.73)$ & $6(5.8)$ & $13(6.3)$ \\
\hline Student & $33(31.73)$ & $22(21.4)$ & $55(26.6)$ \\
\hline Other & $2(1.92)$ & $5(4.8)$ & $7 \quad(3.3)$ \\
\hline \multicolumn{4}{|l|}{ Education completed at what age? } \\
\hline Not yet finished & $27(25.9)$ & $16(15.5)$ & $43(20.8)$ \\
\hline$\leq 16$ years & $24(23.0)$ & $25(24.3)$ & $49(23.7)$ \\
\hline $17+$ years & $53(51.1)$ & $62(60.2)$ & $115(55.5)$ \\
\hline \multicolumn{4}{|l|}{ Smoking (self-description) } \\
\hline Never smoked & $35(33.7)$ & $37(36.6)$ & $72(35.1)$ \\
\hline Ex-smoker & $9(8.7)$ & $18(17.8)$ & $27(13.2)$ \\
\hline Smoking occasionally & $28(26.9)$ & $19(18.8)$ & $47(22.9)$ \\
\hline Regular smoking & $32(30.7)$ & $27(26.8)$ & $59(28.8)$ \\
\hline \multicolumn{4}{|l|}{ Reason for first attendance (GUM) } \\
\hline Asymptomatic screen & $50(50.0)$ & $45(44.6)$ & $95(47.3)$ \\
\hline Symptoms & $46(46.0)$ & $50(49.5)$ & $96(47.7)$ \\
\hline Contact of STI & $4(4.0)$ & $5(5.0)$ & $9(4.5)$ \\
\hline Other & $0 \quad(0.0)$ & $1(0.9)$ & $1(0.5)$ \\
\hline \multicolumn{4}{|l|}{ Alcohol drinking behaviour } \\
\hline \multicolumn{4}{|l|}{ How often having an alcohol drink? } \\
\hline$\leq$ Monthly & $5(4.9)$ & 7 (6.8) & $12(5.8)$ \\
\hline 2-4 Times monthly & $42(40.8)$ & $36(34.9)$ & $78(37.9)$ \\
\hline 2+ Weekly & $56(54.3)$ & $60(58.3)$ & $116(56.3)$ \\
\hline \multicolumn{4}{|l|}{ How many units on a typical day? } \\
\hline $1-2$ & $14(13.6)$ & $12(11.7)$ & $26(12.6)$ \\
\hline $3-6$ & $58(56.3)$ & $51(49.5)$ & $109(52.9)$ \\
\hline $7+$ & $31(30.1)$ & $40(38.8)$ & $71(34.5)$ \\
\hline \multicolumn{4}{|c|}{ How often having $6+$ or $8+$ units on a single occasion? } \\
\hline Never & $1(1.0)$ & $0 \quad(0.0)$ & $1(0.5)$ \\
\hline$\leq$ Monthly & $61(59.2)$ & $59(57.3)$ & $120(58.3)$ \\
\hline Weekly, almost daily or daily & $41(39.8)$ & $44(42.7)$ & $85(41.2)$ \\
\hline AUDIT score & $10.19 \pm 3.9(10,7-13)$ & $9.95 \pm 3.3(10,7-12)$ & $10.1 \pm 3.6(10,7-12)$ \\
\hline Total weekly alcohol units consumed? & $24.95 \pm 26.0(17,6-36)$ & $22.14 \pm 18.8(19,9-30)$ & $23.54 \pm 22.7(18,8-31)$ \\
\hline \multicolumn{4}{|l|}{ Hazardous or harmful drinking $\dagger$} \\
\hline Yes & $55(54.5)$ & $60(58.3)$ & $115(56.4)$ \\
\hline No & $46(45.5)$ & $43(41.7)$ & $89(43.6)$ \\
\hline Total units on the heaviest day & $12.5 \pm 11.3(10,5-16)$ & $12.1 \pm 8.7(10,6-16)$ & $12.3 \pm 10.0(10,6-16)$ \\
\hline
\end{tabular}


Table 1 Continued

\begin{tabular}{|c|c|c|c|}
\hline Parameters* & Usual care $(n=104)$ & Brief intervention $(n=103)$ & Total $(n=207)$ \\
\hline \multicolumn{4}{|l|}{ Drink with an intention of getting drunk? } \\
\hline$\leq$ Monthly & $8(21.6)$ & $5(14.3)$ & $13(18.1)$ \\
\hline 2-4 Times monthly & $7(18.9)$ & $8(22.8)$ & $15(20.8)$ \\
\hline 2+ Weekly & $17(46.0)$ & $17(48.6)$ & $34(47.2)$ \\
\hline 4+ Weekly & $5(13.5)$ & $5(14.3)$ & $10(13.9)$ \\
\hline \multicolumn{4}{|l|}{ Advice on alcohol use } \\
\hline Yes & $68(65.4)$ & $66(64.1)$ & $134(64.7)$ \\
\hline No & $36(34.6)$ & $37(35.9)$ & $73(35.3)$ \\
\hline \multicolumn{4}{|l|}{ Sexual behaviour in last 4 weeks } \\
\hline How many occasions of sex? & $7.0 \pm 8.5(4,1-10)$ & $5.6 \pm 7.7(2,1-10)$ & $6.3 \pm 8.1(3,1-10)$ \\
\hline How many partners? & $1.3 \pm 0.9(1,1-2)$ & $1.3 \pm 1.3(1,1-1)$ & $1.3 \pm 1.1(1,1-2)$ \\
\hline How many were completely new partners? & $0.7 \pm 1.0(0,0-1)$ & $0.7 \pm 1.0(0,0-1)$ & $0.7 \pm 1.0(0,0-1)$ \\
\hline \multicolumn{4}{|l|}{ Condom use with new partners } \\
\hline Yes & $14(40.0)$ & $9(27.3)$ & $23(33.8)$ \\
\hline No & $7(20.0)$ & $9(27.3)$ & $16(23.5)$ \\
\hline Not stated & $14(40.0)$ & $15(45.6)$ & $29(42.7)$ \\
\hline \multicolumn{4}{|l|}{ Drunk and sex that was regretted the next day } \\
\hline Yes & $12(13.3)$ & 8 (8.6) & $20(10.9)$ \\
\hline No & $78(86.7)$ & $85(91.4)$ & $163(89.1)$ \\
\hline
\end{tabular}

*Continuous variables are presented by mean \pm SD (median, IQR); categorical variables are presented by frequency and percentage in parentheses $[n(\%)]$. $\dagger>14$ units/week in women; $>21$ units/week in men.

AUDIT, Alcohol Use Disorders Identification Test; GUM, genitourinary medicine; IQR, interquartile range; STI, sexually transmitted infection.

heavy drinkers and did not have major issues with alcohol consumption. All of the nurses individualised the intervention for patients, rather than delivering every element of BI to each person. The SHC was considered an appropriate environment to deliver BI because of the links between alcohol intake and sexual behaviour. The majority of nurses felt that they were time-constrained and short-staffed in the clinic and did not think they would be able to deliver the intervention long-term without additional resources.

All staff agreed that they were now more informed about units of alcohol from using the unit calculators (tools that work out number of alcohol units in a drink). They believed that the patients also appreciated such tools. Staff suggested that BI may have been easier if it was computer-based, particularly if the programme could aid automatic addition of units, and provide low-, medium- or high-risk guidelines from the patient's data to print and give to patients. Staff felt that more health promotion advertising in the form of DVDs/posters/leaflets in the waiting room would be useful. All agreed that there were no ethical dilemmas in delivering the BI within their consultations.

\section{DISCUSSION}

This study showed that it is feasible to systematically assess alcohol use in a busy SHC, to recruit to an RCT and for staff to be trained and deliver BI. It reinforces the previous observation that alcohol misuse is common in a SHC setting; 14\% were drinking at hazardous or harmful levels, and attendees were a predominantly young group with mean age of 25 years. ${ }^{8}$

It is feasible to screen for alcohol misuse in the SHC waiting room where patients often spend long periods waiting before they are seen by a health care professional. It would be difficult to identify problem drinkers in the absence of such systematic screening in a SHC. Unlike previous studies, ${ }^{18} \mathrm{BI}$ was integrated into the sexual health consultation and delivered by the health care professional seeing the patient. There is evidence from primary care that delivering an intervention immediately after screening is more beneficial than delaying it until a subsequent occasion. ${ }^{19}$ In emergency care, it has been shown that minimising the delay between screening and intervention is more effective, and that identifying alcohol-related risk or harm represents a 'teachable moment' where the patient is potentially more receptive to advice and feedback. ${ }^{20}$ Similarly, making a link between alcohol consumption and the presenting sexual health problem may help patients appreciate the link between alcohol misuse and risky sexual behaviour. It is interesting in our study that just under $50 \%$ of participants were attending with a suspected STI. Previous studies have shown that referring hazardous drinkers to external specialist services is unsatisfactory to patients and is likely to result in a poorer uptake. ${ }^{21}$

Baseline AUDIT scores were reasonably balanced in both the groups with a mean of 10 . However, the lower quartile group had a cut-off score of about 7 , which indicates low-risk drinking. The AUDIT-C used as a 
Table 2 Changes in alcohol behaviour in responders at 6 months $(n=98)$

\begin{tabular}{|c|c|c|c|c|c|c|}
\hline \multirow[b]{2}{*}{ Alcohol drinking behaviour* } & \multicolumn{3}{|l|}{ Usual care $(n=52)$} & \multicolumn{3}{|c|}{ Brief intervention $(n=46)$} \\
\hline & Baseline & At 6 weeks & At 6 months & Baseline & At 6 weeks & At 6 months \\
\hline \multicolumn{7}{|l|}{ How often having an alcohol drink? } \\
\hline$\leq$ Monthly & 1 (1.9) & $4 \quad(9.8)$ & $8(15.4)$ & $4 \quad(8.7)$ & $5 \quad(15.2)$ & $6(13.0)$ \\
\hline 2-4 Times monthly & $19(37.3)$ & $13(31.7)$ & $24(46.2)$ & $15(32.6)$ & $13(39.4)$ & $17(36.9)$ \\
\hline 2+Weekly & $31(60.8)$ & $24 \quad(58.5)$ & $20(38.4)$ & $27(58.7)$ & $15(45.4)$ & $23 \quad(50.1)$ \\
\hline \multicolumn{7}{|l|}{ How many units on a typical day? } \\
\hline $1-2$ & $6(11.8)$ & $2 \quad(4.9)$ & $5(9.8)$ & $6(13.0)$ & $3 \quad(9.4)$ & $3 \quad(6.5)$ \\
\hline $3-6$ & $27(52.9)$ & $16(39.0)$ & $14(27.5)$ & $25(54.3)$ & $13(40.6)$ & $21 \quad(45.7)$ \\
\hline $7+$ & $18(35.3)$ & $23(56.1)$ & $32(62.7)$ & $15(32.7)$ & $16(50.0)$ & $22(47.8)$ \\
\hline \multicolumn{7}{|l|}{$\begin{array}{l}\text { How often having } 6+\text { or } 8+\text { units on } \\
\text { a single occasion? }\end{array}$} \\
\hline Never & - & $1 \quad(2.4)$ & $2(3.9)$ & - & $1 \quad(3.0)$ & $2 \quad(4.4)$ \\
\hline$\leq$ Monthly & $26(50.9)$ & $24 \quad(58.5)$ & $32(61.5)$ & $29(63.0)$ & $22(66.7)$ & $32(71.1)$ \\
\hline Weekly, almost daily or daily & $25(49.1)$ & $16(39.1)$ & $18(34.6)$ & $17(37.0)$ & $10 \quad(30.3)$ & $11(24.5)$ \\
\hline AUDIT score & $10.59 \pm 3.8(11,8-13)$ & - & $9.31 \pm 3.7(9,6-12)$ & $9.37 \pm 3.2(9,7-12)$ & - & $8.89 \pm 3.3(9,7-11)$ \\
\hline Total weekly alcohol units consumed? & $30.62 \pm 28.0(24,9-46)$ & $19.04 \pm 18.2(14,7-28)$ & $17.43 \pm 17.1(15,0-26)$ & $19.71 \pm 14.2(18,9-29)$ & $17.74 \pm 15.9(13,5-26)$ & $17.66 \pm 19.1(13,2-25)$ \\
\hline \multicolumn{7}{|l|}{ Hazardous or harmful drinking $\dagger$} \\
\hline Yes & $32(61.5)$ & $19(46.3)$ & $24(46.2)$ & $24(52.2)$ & $14(42.4)$ & $19(42.2)$ \\
\hline No & $20(38.5)$ & $22(53.7)$ & $28(53.8)$ & $22(47.8)$ & $19(57.6)$ & $26(57.8)$ \\
\hline Total units on the heaviest day & $14.2 \pm 12.6(12,6-19)$ & $9.57 \pm 6.9(10,4-14)$ & $9.96 \pm 9.3(8,0-16)$ & $11.4 \pm 6.8(11,7-16)$ & $10.4 \pm 7.8(11,4-16)$ & $9.8 \pm 9.8(9,2-14)$ \\
\hline \multicolumn{7}{|l|}{ Drink with an intention of getting drunk? } \\
\hline$\leq$ Monthly & $3(16.7)$ & $1 \quad(9.1)$ & $11(21.2)$ & $2(12.5)$ & $1(12.5)$ & $13(28.3)$ \\
\hline 2-4 Times monthly & $4(22.2)$ & 7 (63.6) & $19(36.5)$ & $3(18.7)$ & $3(37.5)$ & $18 \quad(39.1)$ \\
\hline 2+Weekly & $8(44.4)$ & $2(18.2)$ & $21(40.4)$ & $9(56.3)$ & $2(25.0)$ & $12(26.1)$ \\
\hline 4+Weekly & $3(16.7)$ & $1 \quad(9.1)$ & 1 (1.9) & $2(12.5)$ & $2(25.0)$ & $3 \quad(6.5)$ \\
\hline \multicolumn{7}{|l|}{ Advice on alcohol use } \\
\hline Yes & $35(67.3)$ & - & 1 (4.6) & $29(63.0)$ & - & $\begin{array}{ll}0 & (0.0)\end{array}$ \\
\hline No & $17(32.7)$ & $11(100.0)$ & $21(95.4)$ & $17(37.0)$ & $8(100.0)$ & $17(100.0)$ \\
\hline
\end{tabular}

${ }^{*}$ Continuous variables are presented by mean \pm SD (median, IQR); categorical variables are presented by frequency and percentage in parentheses $[n(\%)]$.

$\dagger>14$ units/week in women, $>21$ units/week in men.

AUDIT, Alcohol Use Disorders Identification Test; IQR, interquartile range.

$\overrightarrow{6}$ 


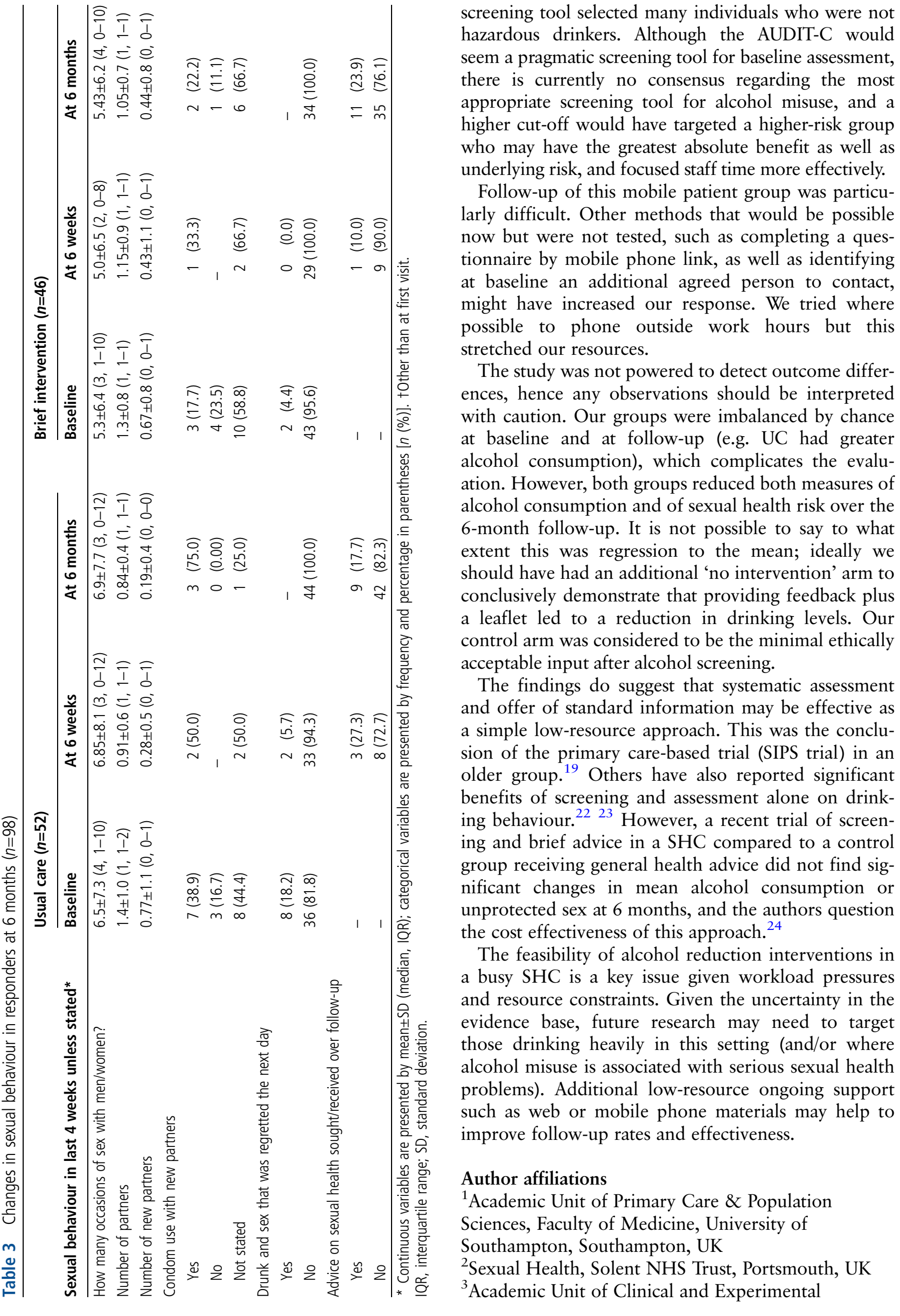


Sciences, Faculty of Medicine, University of

Southampton, Southampton, UK

${ }^{4}$ South Central Strategic Health Authority, Reading, UK

Acknowledgements The authors are very grateful to all the patients who took part in the study and all the clinic staff who helped to run the study smoothly. They would like to specifically thank the Portsmouth City Alcohol Intervention team for providing the training, Jo Buglass who undertook many of the follow-up phone interviews and Dr Esther Mugweni, Mr Scott Harris and Judy Chatwin for help in setting up the study.

Funding National Institute for Health Research (NIHR) Research for Patient Benefit Programme (RfPB) South Central (Reference No. PB-PG-1208-18156).

Competing interests None declared.

Ethics approval Southampton and South West Hampshire Research Ethics Committee 10/H0502/76.

Provenance and peer review Not commissioned; externally peer reviewed.

\section{REFERENCES}

1 Department of Health. On the State of the Public Health. The Annual Report of the Chief Medical Officer of the Department of Health 2001. 2001. http://webarchive.nationalarchives.gov. uk/20130107105354/http://www.dh.gov.uk/prod_consum_dh/ groups/dh_digitalassets/@dh/@en/documents/digitalasset/dh_ 4082273.pdf [accessed 26 December 2013].

2 Health and Social Care Information Centre. Statistics on Alcohol - England, 2013. 2013. http://www.hscic.gov.uk/ catalogue/PUB10932 [accessed 26 December 2013].

3 Leon DA, McCambridge J. Liver cirrhosis mortality rates in Britain 1950-2002. Lancet 2006;367:645.

4 Health and Social Care Information Centre. Adult Psychiatric Morbidity in England - 2007, Results of a Household Survey. 2009. http://www.hscic.gov.uk/catalogue/PUB02931 [accessed 26 December 2013].

5 Health Protection Agency. Sexually Transmitted Infections and Young People in the United Kingdom: 2008 Report. http://www. hpa.org.uk/Publications/InfectiousDiseases/HIVAndSTIs/ 0807STIsyoungpeopleinUK2008/ [accessed 26 December 2013].

6 Maisto SA, Cavey MP, Cavey KB, et al. The relationship between alcohol and individual differences variables on attitudes and behavioural skills relevant to sexual health among heterosexual young adult men. Arch Sex Behav 2004;33:571-584.

7 Cook RL, Clark DB. Is there an association between alcohol consumption and sexually transmitted diseases? A systematic review. Sex Transm Dis 2003;32:156-164.

8 Standerwick K, Davies C, Tucker L, et al. Binge drinking, sexual behaviour and sexually transmitted infection in the UK. Int J STD AIDS 2007;18:810-813.

9 Kaner EF, Beyer F, Dickinson HO, et al. Effectiveness of brief alcohol interventions in primary care populations. Cochrane Database Syst Rev 2007;2:CD004148.
10 Lane J, Proude EM, Conigrave KM, et al. Nurse-provided screening and brief intervention for risky alcohol consumption by sexual health clinic patients. Sex Transm Infect 2008;84:524-527.

11 National Institute of Health and Care Excellence (NICE). Alcohol-use Disorders: Preventing Harmful Drinking (NICE Guideline PH24). London, UK: NICE, 2010. http://www.nice. org.uk/ph24 [accessed 26 December 2013].

12 Gual A, Segura L, Contel M, et al. Audit-3 and Audit-4: effectiveness of two short forms of the alcohol use disorders identification test. Alcohol Alcohol 2002;37:591-596.

13 Saunders JB, Aasland OG, Babor TF, et al. Development of the Alcohol Use Disorders Identification Test (AUDIT): WHO collaborative project on early detection of persons with harmful alcohol consumption-II. Addiction 1993;88:791-804.

14 Reinert DF, Allen JP. The alcohol use disorders identification test: an update of research findings. Alcohol Clin Exp Res 2007;31:185-199.

15 Miller WR, Sanchez VC. Motivating young adults for treatment and lifestyle change. In: Howard GS, Nathan PE (eds). Alcohol Use and Misuse by Young Adults. South Bend, IN: University of Notre Dame Press, 1993;55-82.

16 Miller WR, Zweben A, DiClemente CC, et al. Motivational Enhancement Therapy Manual: A Clinical Research Guide for Therapists Treating Individuals with Alcohol Abuse and Dependence (Project MATCH Monograph Series, Vol. 2). Rockville, MD: National Institute on Alcohol Abuse and Alcoholism (NIAAA), 1992.

17 Braun V, Clarke V. Using thematic analysis in psychology. Qual Res Psychol 2006;3:77-101.

18 Crawford MJ, Lowe PC, Greene L, et al. The prevalence of excessive alcohol consumption and the acceptability of brief advice in a sexual health clinic: cross sectional survey. Sex Trans Inf 2004;80:416-417.

19 Kaner E, Bland M, Cassidy P, et al. Effectiveness of screening and brief alcohol intervention in primary care (SIPS trial): pragmatic cluster randomised controlled trial. BMJ 2013;346: e8501.

20 Williams S, Brown A, Patton B, et al. The half-life of the "teachable moment" for alcohol misusing patients in the emergency department. Drug Alcohol Depend 2005;77:205-208.

21 Thorley N, Hettiarachchi N, Nightingale P, et al. Screening for hazardous alcohol consumption in a sexual health clinic: a service evaluation. Int J STD AIDS 2012;23:585-588.

22 McCambridge J, Day M. Randomised controlled trial of the effects of completing the Alcohol Use Disorders Identification Test questionnaire on self-reported hazardous drinking. Addiction 2007;103:241-248.

23 Kypri K, Langley JD, Saunders JB, et al. Assessment may conceal therapeutic benefit: findings from a randomised controlled trial for hazardous drinking. Addiction 2007;102:62-70.

24 Crawford MJ, Sanatiana R, Barrett B, et al. The clinical and cost-effectiveness of brief advice for excessive alcohol consumption among people attending sexual health clinics: a randomised controlled trial. Sex Trans Inf 2015;91:37-43. 